\title{
COVID-19-Induced Hepatic Injury: A Systematic Review and Meta-Analysis
}

\author{
Sara Abdulla ${ }^{1}$, Azhar Hussain ${ }^{2,3}$, Dua Azim ${ }^{4}$, Enas H. Abduallah ${ }^{5}$, Hayam Elawamy ${ }^{6}$, Sundus Nasim ${ }^{4}$, \\ Sohail Kumar ${ }^{4}$, Hassan Naveed ${ }^{7}$ \\ 1. Biochemistry, University of Benghazi, Benghazi, LBY 2. Healthcare Administration, Franklin University, Columbus, \\ USA 3. Medicine, Xavier University School of Medicine, Oranjestad, ABW 4. Internal Medicine, Dow Medical College, \\ Dr. Ruth K. M. Pfau Civil Hospital, Karachi, PAK 5. Mathematics, University of Tobruk, Tobruk, LBY 6. Medical \\ Laboratory, College of Medical Technology, Benghazi, LBY 7. Internal Medicine, St. Matthew's University School of \\ Medicine, Grand Cayman, CYM
}

Corresponding author: Dua Azim, duaazeem456@gmail.com

\section{Abstract \\ Background}

The current pandemic of the novel coronavirus disease (COVID-19) is a global health challenge. Pulmonary dysfunction is the main outcome of COVID-19 infection. In critically ill patients, however, liver complications have also been reported. Thus, we conducted a systematic review and meta-analysis to draw generalized conclusions regarding impaired liver biochemistry and its potential relationship with COVID-19 disease severity.

\section{Materials and Methods}

We searched the PubMed, Scopus, and Web of Science databases for all the related literature published up to June 20, 2020. The data were analyzed using R statistical software. A random-effects model was employed for pooling the data. The risk of bias and quality of included studies was assessed using the modified NewcastleOttawa Scale (NOS) for cohort studies.

\section{Results}

The present meta-analysis comprises 10 retrospective and two prospective studies (6,976 COVID-19 patients). The serum analysis revealed significantly higher levels of alanine aminotransferases and aspartate aminotransferases and significantly lower albumin levels. Moreover, insignificant increases in serum levels of total bilirubin were observed. Upon subgroup analysis of six studies (severe cases, $n=131$; non-severe cases, $\mathrm{n}=334$ ) stratified on the basis of disease severity, we found that these abnormalities were relatively higher in severe cases of COVID-19 (albumin [weighted mean difference (WMD), 34.03 g/L; 95\% CI, 27.42 to 40.63; $\mathrm{p}<0.0001 ; \mathrm{I}^{2}=96.83 \%$ ); alanine transaminase (ALT) [WMD, $31.66 \mathrm{U} / \mathrm{L} ; 95 \%$ CI, 25.07 to 38.25; $\mathrm{p}<0.0001 ; \mathrm{I}^{2}=55.64 \%$ ]; aspartate aminotransferase (AST) [WMD, $41.79 \mathrm{U} / \mathrm{L} ; 95 \% \mathrm{CI}, 32.85$ to $50.72 ; \mathrm{p}<0.0001$; $\mathrm{I}^{2}=51.43 \%$ ]; total bilirubin [WMD, $9.97 \mu \mathrm{mol} / \mathrm{L} ; 95 \% \mathrm{CI}$, 8.46 to $11.48 ; \mathrm{p}<0.0001 ; \mathrm{I}^{2}=98 \%$ ]) than in non-severe cases.

Review began 09/29/2020 Review ended 10/08/2020 Published 10/13/2020

\section{() Copyright 2020}

Abdulla et al. This is an open access article distributed under the terms of the Creative Commons Attribution License CC-BY 4.0., which permits unrestricted use, distribution, and reproduction in any medium, provided the original author and source are credited.

\section{Conclusion}

Deranged liver enzymes serve as prognostic factors to assess the severity of COVID-19. Liver markers should, therefore, be observed and monitored continuously.

Categories: Internal Medicine, Gastroenterology, Infectious Disease

Keywords: sars-cov-2, covid-19, hepatic injury, liver injury, liver injury biomarkers, lfts, systematic review and metaanalysis

\section{Introduction}

In early December 2019, the novel coronavirus disease 2019 (COVID-19) was first identified in Wuhan City, China, as a cluster of rare cases of pneumonia [1]. Since then, the highly contagious COVID-19, caused by severe acute respiratory syndrome coronavirus 2 (SARS-CoV-2), has spread globally, causing substantial morbidity and mortality. With approximately 118,000 cases and 4,291 deaths recorded worldwide, this disease was declared a pandemic by the World Health Organization (WHO) on March 11, 2020. By June 30, 2020 , a total of $10,185,374$ confirmed cases and 503,862 deaths were documented in 216 countries [2].

The typical presentation of COVID-19 involves fever, weakness, nausea, and symptoms of pulmonary distress such as dry cough and dyspnea. However, the understanding and knowledge of the disease have improved over time, and it has become evident that SAR-CoV-2 damages not only the respiratory system but also the cardiovascular, gastrointestinal, and hepatobiliary systems, subsequently resulting in multi-organ 


\section{Cureus}

failure (MOF) and death [3-4].

The involvement of the liver was also seen in the Middle East respiratory syndrome coronavirus (MERS-CoV) and SARS-CoV. Owing to their remarkable genetic similarity with SARS-CoV-2, liver involvement in COVID-19 was already predicted [5]. While the exact cause of hepatic injury is uncertain, the following major mechanisms have been suggested: (i) direct injury to hepatocytes or biliary epithelium; (ii) druginduced hepatoxicity; (iii) liver injury related to exaggerated defense response of the body; and (iv) exacerbation of hepatic dysfunction by COVID-19 in individuals suffering from pre-existing liver disorders [6].

To date, however, literature regarding the correlation of COVID-19 with liver dysfunction has been minimal. Given the limited data, we aim to perform a meta-analysis and systematically review the current data available on liver injury in COVID-19 with two main objectives: (i) to draw more generalized conclusions about the abnormal serum markers of liver injury such as albumin, alanine aminotransferase (ALT), aspartate aminotransferase (AST), and bilirubin in laboratory-confirmed COVID-19 patients; and (ii) to determine its relationship with the severity of COVID-19.

\section{Materials And Methods}

\section{Literature search strategy}

We conducted this meta-analysis as per the guidelines provided by Preferred Reporting Items for Systematic Reviews and Meta-Analyses (PRISMA). The authors independently searched the Medline (PubMed interface), Scopus, and Web of Science databases using the keywords "COVID-19" or "2019-nCoV" and "laboratory data of Coronavirus infection” for all related publications up till June 20, 2020. The bibliography of relevant articles was scanned for any missed qualified paper. The electronic search strategy for all three databases is detailed in Table 1.

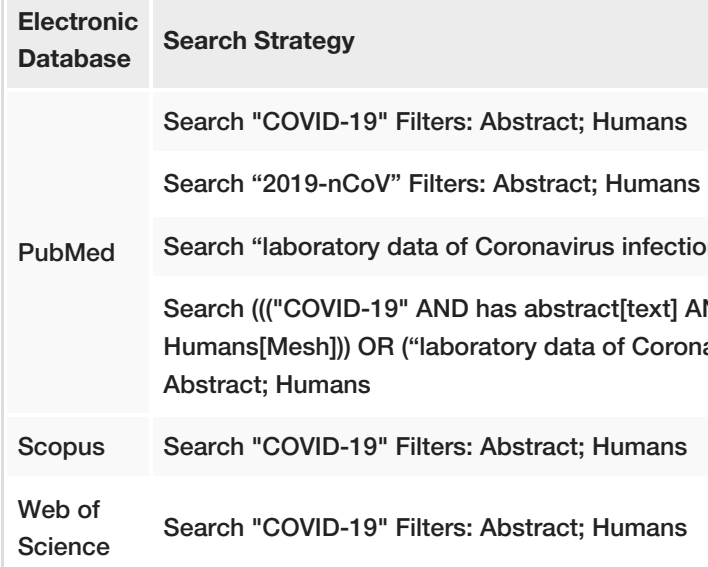

TABLE 1: Search strategy of electronic databases

\section{Criteria for liver injury and disease severity}

The present analysis involved adults with COVID-19 and associated liver damage, irrespective of their preexisting chronic liver disease or COVID-19 severity. We described the liver injury as having serum alanine transaminase (ALT) and aspartate aminotransferase (AST) level $>50 \mathrm{U} / \mathrm{L}$ and $>40 \mathrm{U} / \mathrm{L}$, respectively. Hypoalbuminemia was identified as serum albumin level $<40 \mathrm{~g} / \mathrm{L}$; total bilirubin level $>21 \mathrm{mmol} / \mathrm{L}$ was regarded as hyperbilirubinemia. Additionally, severity was defined according to the need for intensive care unit (ICU) admission, need for oxygen support, or death, or in parallel to the criteria explained in the studies.

\section{Study selection}

All extracted articles were tested for their eligibility based on the following inclusion criteria: (i) studies reporting reverse transcription-polymerase chain reaction (RT-PCR)-confirmed COVID-19 cases; (ii) reported studies of liver biomarkers (albumin, bilirubin, ALT, AST) and their mean serum levels among severe and non-severe cases of COVID-19; (iii) studies mentioning most of the laboratory data quantitatively, not qualitatively; and (iv) studies containing the characteristics and demographic information of the patients along with the year, country, number of patients, age, and sex. 
studies reporting COVID-19 patients without laboratory diagnosis; (iii) case reports, case series, or any study having a sample size of less than 10; (iv) studies that lack relevant information for any reason; (v) papers that did not include primary information such as reviews, consensus, and guidelines; and (vi) studies involving pregnant women and children.

\section{Data extraction and quality assessment}

Initially, titles and abstracts were analyzed for inclusion criteria; the full text was examined in cases where the abstract was inadequate to assess whether the study met the inclusion criteria. For all eligible articles, data such as first author, year of publication, location, number of patients, age, sex, and serum levels of liver biomarkers (albumin, AST, ALT, and bilirubin) were extracted and recorded. The Microsoft Excel database (Microsoft Corporation, Redmond, Washington) was used to record all available laboratory data. Inconsistencies between the researchers were discussed to reach consensus.

The modified Newcastle-Ottawa Scale (NOS) for cohort studies was used to evaluate the quality and risk of bias of eligible papers. Studies with a NOS score of $\geqslant 5,3-4,<3$ were regarded as high, medium, and lowquality publications, respectively. We used the GRADEpro software (McMaster University, 2020, Hamilton, Canada) to assess the quality of evidence and graded it as high, moderate, low, and very low.

\section{Statistical analysis}

R statistical software (version 3.6.1; R Foundation for Statistical Computing, Vienna, Austria) was used to conduct statistical analysis. Additionally, the meta-package was employed to measure the proportion of COVID-19 positive individuals with deranged liver function tests (LFTs). First, we unified all the units of variables; we then expressed classified variables as percentages and continuous variables as median and inter-quartile range (IQR). Studies' heterogeneity was assessed using Higgin's I-square $\left(\mathrm{I}^{2}\right)$ test; $\mathrm{I}^{2}$ values of $0-25 \%, 25-50 \%, 50-75 \%$, and $>75 \%$ were indicated as insignificant, low, moderate, and high heterogeneity, respectively. We used the random-effect model for calculating the pooled median with a $95 \%$ confidence interval (CI) if $\mathrm{I}^{2} \geqslant 50 \%$; the fixed-effect model was selected if $\mathrm{I}^{2}<50 \%$.

A meta-analysis of variations in serum levels of albumin, AST, ALT, and total bilirubin was not carried out, as the selected studies presented the median values of lactate dehydrogenase (LDH) only; the reference ranges also varied among the studies due to different detection methods. Thus, a meta-analysis was conducted separately for each group of non-severe and severe patients and then compared.

\section{Results}

\section{Study selection process}

The initial search yielded 789 articles. After removing 409 duplicates, a total of 380 articles were examined for title and abstract. Moreover, another 30 studies were excluded, as they specifically involved pregnant women and children. Thus, 350 papers were eligible for full-text screening. A total of 338 articles were excluded after reading the full text due to various reasons. Eventually, 12 studies, with a total number of 6,976 COVID-19 patients, were included in this systemic review and meta-analysis [1,3,7-16]. The study selection process using the PRISMA flow diagram is shown in Figure 1. 


\section{Cureus}

PRISMA 2009 Flow Diagram

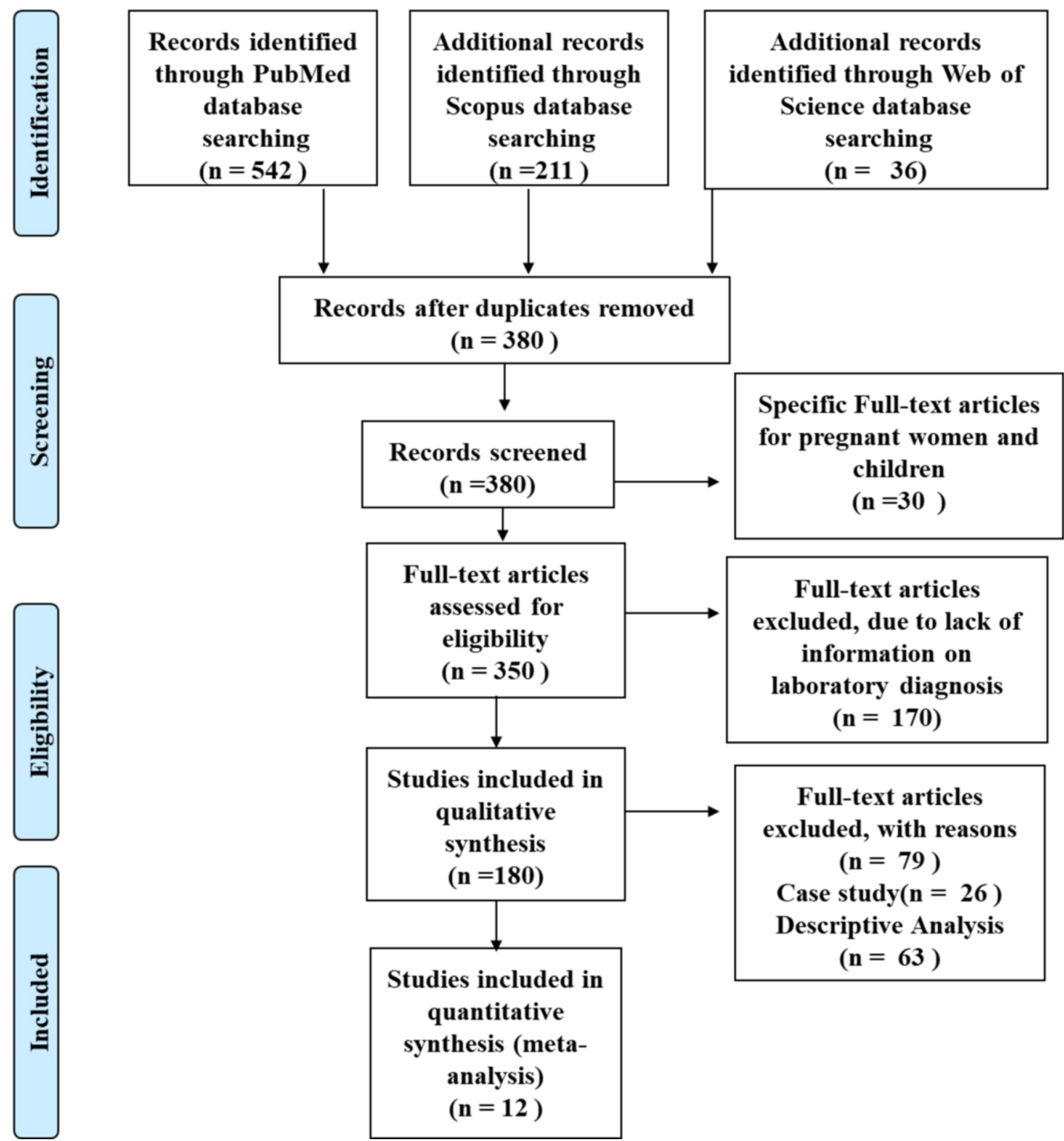

FIGURE 1: PRISMA flowchart showing the study selection process for the meta-analysis

PRISMA: Preferred Reporting Items for Systemic Reviews and Meta-Analyses

\section{Study characteristics}

Of the 12 studies, 10 had a retrospective [1,7-11,13-16] and two had a prospective study design [3,12]. Nine studies were from China while the remaining three were from the United States of America (USA), Italy, and Oman. Four studies reported multicenter data while the remaining eight studies reported single-center data. The sample size of studies varied between 21 and 5,700 patients with a mean age of 50.9 years (age range, $21-95$ years). The results of the analysis demonstrated a male-dominant pattern; $60.2 \%$ of all the patients were male while the remaining $39.8 \%$ were females. All 12 studies were conducted in a hospital setting. The laboratory tests were obtained at the time of admission in each study included in the present meta-analysis. The characteristics of the studies enrolled in this analysis are listed in Table 2. 


\section{Cureus}

\begin{tabular}{|c|c|c|c|c|c|c|c|c|c|}
\hline \multirow{2}{*}{ Author } & \multirow{2}{*}{ Year } & \multirow{2}{*}{ Country } & \multirow{2}{*}{ Study Design } & \multirow{2}{*}{$\begin{array}{l}\text { Sample } \\
\text { Size }\end{array}$} & Age, years & Female & Male & $\begin{array}{l}\text { Baseline } \\
\text { CLD }\end{array}$ & $\begin{array}{l}\text { Follow-up } \\
\text { Time }\end{array}$ \\
\hline & & & & & Median (IQR) & n (\%) & n (\%) & (\%) & (Days) \\
\hline Wan S et al. [14] & 2020 & China & Retrospective & 135 & 47 (36-55) & $63(46.7)$ & $72(53.3)$ & 1.48 & 16 \\
\hline Chen $\mathrm{N}$ et al. [1] & 2020 & China & Retrospective & 99 & $55.5(21-82)^{\mathrm{a}}$ & 32 (32) & $67(68)$ & NR & 20 \\
\hline Jin $X$ et al. [10] & 2020 & China & Retrospective & 74 & $46.14 \pm 14.19^{b}$ & $37(50)$ & $37(50)$ & 3.8 & 23 \\
\hline Feng Y et al. [8] & 2020 & China & Retrospective & 476 & $53(40-64)$ & $205(43)$ & $271(57)$ & NR & $N R$ \\
\hline Huang C et al. [3] & 2020 & China & Prospective & 41 & $49 \cdot 0(41-58)$ & $11(27)$ & $30(73)$ & 2.44 & 32 \\
\hline $\begin{array}{l}\text { Richardson S et al. } \\
\text { [13] }\end{array}$ & 2020 & USA & Retrospective & 5700 & $63(52-75)$ & $\begin{array}{l}2263 \\
(39.7)\end{array}$ & $\begin{array}{l}3437 \\
(60.3)\end{array}$ & 0.52 & 35 \\
\hline Inciardi RM et al. [9] & 2020 & Italy & Retrospective & 99 & $67 \pm 12^{\mathrm{b}}$ & $19(19.2)$ & $80(80.8)$ & NR & 14 \\
\hline Wang D et al. [15] & 2020 & China & Retrospective & 138 & $56(42-68)$ & $63(45.7)$ & $75(54.3)$ & 2.9 & 34 \\
\hline Khamis F et al. [11] & 2020 & Oman & Retrospective & 63 & $48 \pm 16^{b}$ & 10 (15) & $53(85)$ & NR & 61 \\
\hline Liu J et al. [12] & 2020 & China & Prospective & 61 & $40(1-86)$ & $30(49.2)$ & $31(50.8)$ & NR & 10 \\
\hline Wang Z et al. [16] & 2020 & China & Retrospective & 69 & $42(35-62)$ & $37(54)$ & $32(46)$ & 1.45 & 19 \\
\hline Chen G & 20 & ina & trospective & 21 & $6(50-6$ & $4(19)$ & $17(81)$ & NR & NR \\
\hline
\end{tabular}

\section{TABLE 2: Characteristics of the studies included in the meta-analysis}

CLD: chronic liver disease; NR: not reported

aMean (range); ${ }^{\text {bMean } \pm S D}$

\section{Laboratory data}

Regarding the LFTs of COVID-19 positive patients, the most prevalent abnormality was reduced serum albumin level, whereas liver enzymes were mostly normal or marginally raised. Overall mean serum levels for albumin, ALT, AST, and total bilirubin are shown in Table 3. The blood biochemistry parameters for studies stratified based on the severity of COVID-19 are demonstrated in Table 4 [3,7,12,14-16]. 


\section{Cureus}

\begin{tabular}{|c|c|c|c|c|}
\hline \multirow{2}{*}{ Author } & $\begin{array}{l}\text { Albumin }(\mathrm{g} / \mathrm{L} ; \text { normal } \\
\text { range, } 40-55)\end{array}$ & $\begin{array}{l}\text { ALT (U/L; normal } \\
\text { range, 9-50) }\end{array}$ & $\begin{array}{l}\text { AST (U/L; normal } \\
\text { range, 15-40) }\end{array}$ & $\begin{array}{l}\text { Total Bilirubin ( } \mu \mathrm{mol} / \mathrm{L} \text {; normal } \\
\text { range, } 0-21 \text { ) }\end{array}$ \\
\hline & Median (IQR) & Median (IQR) & Median (IQR) & Median (IQR) \\
\hline Wan S et al. [14] & $40.5(37-43.4)$ & 26 (12.9-33.15) & $33.4(27.8-43.7)$ & $8.6(5.9-13.7)$ \\
\hline Chen $\mathrm{N}$ et al. [1] & NR & $39(22-53)$ & $34(26-48)$ & $15 \cdot 1(7 \cdot 3)$ \\
\hline Jin X et al. [10] & $40.13(35.95-42.6)$ & $25(15.75-38.47)$ & 29.35 (20.87-38.62) & 10 (7.15-13.8) \\
\hline Feng Y et al. [8] & $37.87(32.8-41.84)$ & $26(16-41)$ & $28(21-39)$ & $10.1(7.5-14)$ \\
\hline Huang C et al. [3] & $31 \cdot 4(28 \cdot 9-36)$ & $32(21-50)$ & $34(26-48)$ & $11 \cdot 7(9 \cdot 5-13 \cdot 9)$ \\
\hline $\begin{array}{l}\text { Richardson S et } \\
\text { al. [13] }\end{array}$ & NR & $33(21-55)$ & $46(31-71)$ & NR \\
\hline $\begin{array}{l}\text { Inclaardi RM et al. } \\
\text { [9] }\end{array}$ & $33(29.4-36)$ & $34(24-58)$ & $46(34-68)$ & NR \\
\hline $\begin{array}{l}\text { Wang D et al. } \\
\text { [15] }\end{array}$ & NR & $24(16-40)$ & $31(24-51)$ & $9.8(8.4-14.1)$ \\
\hline $\begin{array}{l}\text { Khamis F et al. } \\
{[11]}\end{array}$ & NR & NR & NR & $10(6-14)$ \\
\hline Liu J et al. [12] & $44(50.5-47)$ & $19(14-33.5)$ & NR & NR \\
\hline $\begin{array}{l}\text { Wang Z et al. } \\
\text { [16] }\end{array}$ & NR & $23(17-40)$ & $28(22-42)$ & NR \\
\hline Chen $\mathrm{G}$ et al. [7] & $33.7(29.6-37.4)$ & $26(16-42)$ & $27(21-47)$ & $8.8(6.8-10.3)$ \\
\hline
\end{tabular}

\section{TABLE 3: Liver function tests of the included studies}

ALT: alanine aminotransferase; AST: aspartate aminotransferase; NR: not reported 


\section{Cureus}

\begin{tabular}{|c|c|c|c|c|c|}
\hline \multirow{2}{*}{ Authors } & \multirow{2}{*}{ Severity of Disease } & Albumin & ALT & AST & Total Bilirubin \\
\hline & & Median (IQR) & Median (IQR) & Median (IQR) & Median (IQR) \\
\hline \multirow{2}{*}{ Wan S et al. [14] } & Non-severe $(n=95)$ & $49.9(37.4-43.6)$ & $21.7(14.8-36.9)$ & 22.4 (16.9-30.5) & $8.6(5.6-14)$ \\
\hline & Severe $(n=40)$ & 36.0 (33-38.5) & $26.6(14.5-33.3)$ & $33.6(25.7-44.2)$ & $9.8(7.8-15.6)$ \\
\hline \multirow{2}{*}{ Huang } & Non-severe $(n=28)$ & $34 \cdot 7(30 \cdot 2-36 \cdot 5)$ & $27.0(19 \cdot 5-40)$ & $34.0(24-40.5)$ & $10 \cdot 8(9 \cdot 4-12 \cdot 3)$ \\
\hline & Severe $(n=13)$ & $27 \cdot 9(26 \cdot 3-30 \cdot 9)$ & $49.0(29-115)$ & $44.0(30-70)$ & $14.0(11 \cdot 9-32 \cdot 9)$ \\
\hline \multirow{2}{*}{ Chen G } & Non-severe $(n=10)$ & $37.2(35.8-38.8)$ & $16.0(13.3-21.8)$ & $24.0(21.5-26.5)$ & $7.8(6.4-9.5)$ \\
\hline & Severe $(n=11)$ & $29.6(28.6-33)$ & $42.0(32.5-50)$ & $47.0(28-74.5)$ & $8.8(7.9-10.5)$ \\
\hline \multirow{2}{*}{ Liu J et al. [12] } & Non-severe $(n=44)$ & $44.0(41-47)$ & $18.0(14-32.3)$ & NR & NR \\
\hline & Severe $(n=17)$ & $43.0(37-45.5)$ & $24.0(14-34.5)$ & NR & NR \\
\hline \multirow{2}{*}{ Wang $Z$ et al. [16] } & Non-severe $(n=55)$ & NR & $24(16-40)$ & $26(21-39)$ & NR \\
\hline & Severe $(n=14)$ & NR & $31.5(23-52)$ & $40.5(24-62)$ & NR \\
\hline \multirow{2}{*}{ Wang D et al. [' } & Non-severe $(n=120)$ & NR & $23.0(15-36)$ & $29.0(21-38)$ & $9.3(8.2-12.8)$ \\
\hline & Severe $(n=36)$ & NR & $35.0(19-57)$ & $52.0(30-70)$ & $11.5(9.6-18.6)$ \\
\hline
\end{tabular}

\section{TABLE 4: Liver function tests of the six studies stratified on the basis of severity}

\section{Pooled analysis of laboratory findings}

The pooled proportion of abnormal LFT parameters in COVID-19 positive patients showed significantly

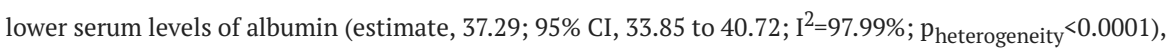
and significantly higher serum levels of AST (estimate, 33.84; 95\% CI, 29.47 to 38.20; $\mathrm{I}^{2}=96.03 \%$;

$\mathrm{p}_{\text {heterogeneity }}<0.0001$ ) and ALT (estimate, 27.93; 95\% CI, 24.57 to 31.30; $\mathrm{I}^{2}=91.35 \%$; $\mathrm{p}_{\text {heterogeneity }}<0.0001$ ). In addition, an insignificant increase in the serum levels of total bilirubin (estimate, 9.87; 95\% CI, 9.14 to 10.59 ; $\mathrm{I}^{2}=66.78 \%$; $\mathrm{p}_{\text {heterogeneity }}<0.0173$ ) was observed. A random-effect method was adopted since the heterogeneity between the studies was high for all four parameters. Pooled estimates of LFTs are demonstrated in Table 5. The individual forest plots for pooled estimates of serum levels of albumin, ALT, AST, and total bilirubin are shown in Figure 2, Figure 3, Figure 4, and Figure 5, respectively. Moreover, upon quality assessment of the outcomes, they were rated to be of high quality. The quality of evidence is outlined in Table 5. 


\section{Cureus}

\begin{tabular}{|c|c|c|c|c|c|c|c|c|c|c|c|}
\hline \multirow{2}{*}{ Variable } & \multirow{2}{*}{$\mathbf{N}^{\mathrm{a}}$} & \multirow{2}{*}{ Estimate } & \multirow{2}{*}{$95 \% \mathrm{Cl}$} & \multirow{2}{*}{$\mathbf{N}^{\mathbf{b}}$} & \multirow{2}{*}{$\begin{array}{l}\text { Standard } \\
\text { Error }\end{array}$} & \multirow{2}{*}{$p$-value } & \multicolumn{4}{|c|}{ Measure of Heterogeneity } & \multirow{2}{*}{$\begin{array}{l}\text { Quality of Evidence } \\
\text { (GRADE) }\end{array}$} \\
\hline & & & & & & & $\mathrm{T}^{2}$ & Q & $\mathrm{p}$ & $\mathrm{I}^{2}$ & \\
\hline Albumin & 7 & 37.29 & $\begin{array}{l}33.85- \\
40.72\end{array}$ & 907 & 1.752 & $<0.0001$ & 20.730 & 207.597 & $<0.0001$ & $97.99 \%$ & $\oplus \oplus \oplus \oplus \mathrm{HIGH}$ \\
\hline ALT & 11 & 27.93 & $\begin{array}{l}24.57- \\
31.30\end{array}$ & 6913 & 1.716 & $<0.0001$ & 25.894 & 120.37 & $<0.0001$ & $91.35 \%$ & $\oplus \oplus \oplus \oplus \mathrm{HIGH}$ \\
\hline AST & 10 & 33.84 & $\begin{array}{l}29.47- \\
38.20\end{array}$ & 6852 & 2.22 & $<0.0001$ & 45.770 & 522.047 & $<0.0001$ & $96.03 \%$ & $\oplus \oplus \oplus \oplus \mathrm{HIGH}$ \\
\hline $\begin{array}{l}\text { Total } \\
\text { Bilirubin }\end{array}$ & 7 & 9.87 & 9.14-10.59 & 948 & 0.3698 & $<0.0001$ & 0.589 & 15.406 & 0.0173 & $66.78 \%$ & $\oplus \oplus \oplus \oplus \mathrm{HIGH}$ \\
\hline
\end{tabular}

\section{TABLE 5: Incidence of abnormal liver function tests: result of meta-analysis}

$\mathrm{CI}$, confidence interval; ALT, alanine aminotransferase; AST, aspartate aminotransferase

$\mathrm{N}^{\mathrm{a}}$, number of studies; $\mathrm{N}^{\mathrm{b}}$, number of patients

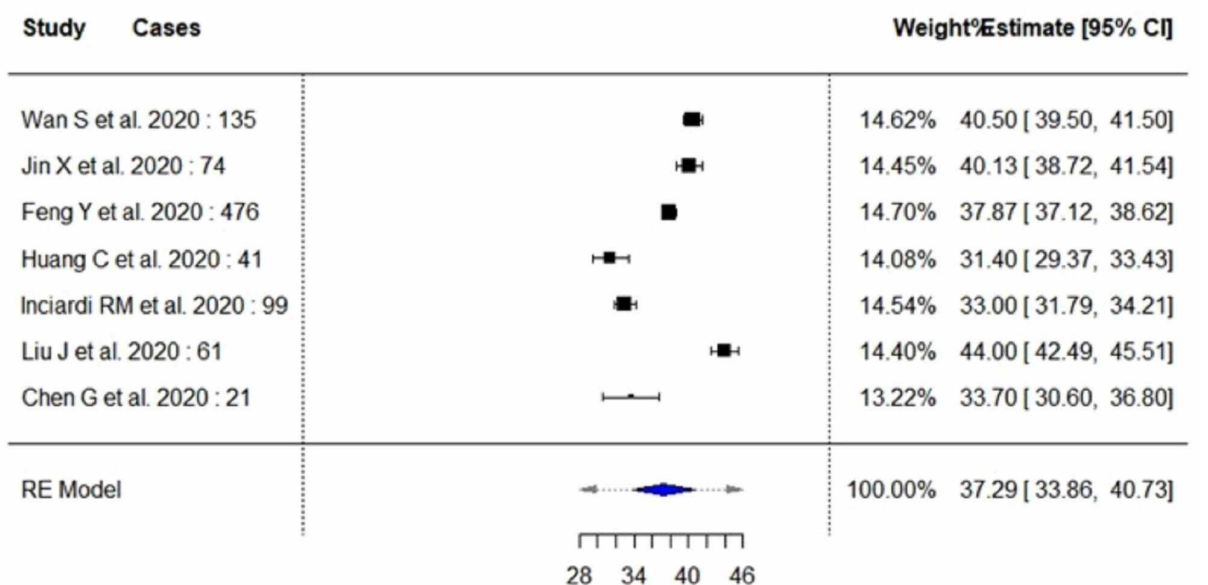

Study weight,Albumin $\& 95 \mathrm{Cl}$

FIGURE 2: Forest plots for pooled analysis of serum levels of albumin using a random-effects model

Cl: confidence interval 


\section{Cureus}

\begin{tabular}{|c|c|c|c|}
\hline Cases & & Weis & htor ${ }^{2}$ stimate $[95 \% \mathrm{Cl}]$ \\
\hline Wan S et al. $2020: 135$ & $\mapsto$ & $10.33 \%$ & $26.00[22.83,29.17]$ \\
\hline Chen $N$ et al. $2020: 99$ & $\longmapsto$ & $8.60 \%$ & $39.00[33.33,44.67]$ \\
\hline Jin X et al. $2020: 74$ & $\longmapsto$ & $9.36 \%$ & $25.00[20.36,29.64]$ \\
\hline Feng $\mathrm{Y}$ et al. $2020: 476$ & $=$ & $10.94 \%$ & $26.00[24.00,28.00]$ \\
\hline Huang $C$ et al. $2020: 41$ & $\longmapsto$ & $6.91 \%$ & $32.00[23.98,40.02]$ \\
\hline Richardson S et al. $2020: 570$ & - & $11.31 \%$ & $33.00[32.21,33.79]$ \\
\hline Inciardi RM et al. $2020: 99$ & $\longmapsto$ & $8.23 \%$ & $34.00[27.84,40.16]$ \\
\hline Wang D et al. 2020 : 138 & $\longmapsto$ & $10.04 \%$ & $24.00[20.36,27.64]$ \\
\hline Liu J et al. $2020: 61$ & $\longmapsto$ & $9.42 \%$ & $19.00[14.45,23.55]$ \\
\hline Wang Z et al. $2020: 69$ & $\longmapsto$ & $9.14 \%$ & $25.00[20.06,29.94]$ \\
\hline Chen G et al. 2020 : 21 & & $5.71 \%$ & $26.00[16.07,35.93]$ \\
\hline RE Model & 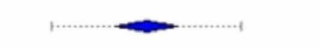 & $100.00 \%$ & $27.94[24.57,31.30]$ \\
\hline & 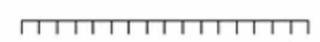 & & \\
\hline & $\begin{array}{llllll}14 & 20 & 26 & 32 & 38 & 44\end{array}$ & & \\
\hline
\end{tabular}

FIGURE 3: Forest plots for pooled analysis of serum levels of ALT using a random-effects model

Cl: confidence interval; ALT: alanine aminotransferase

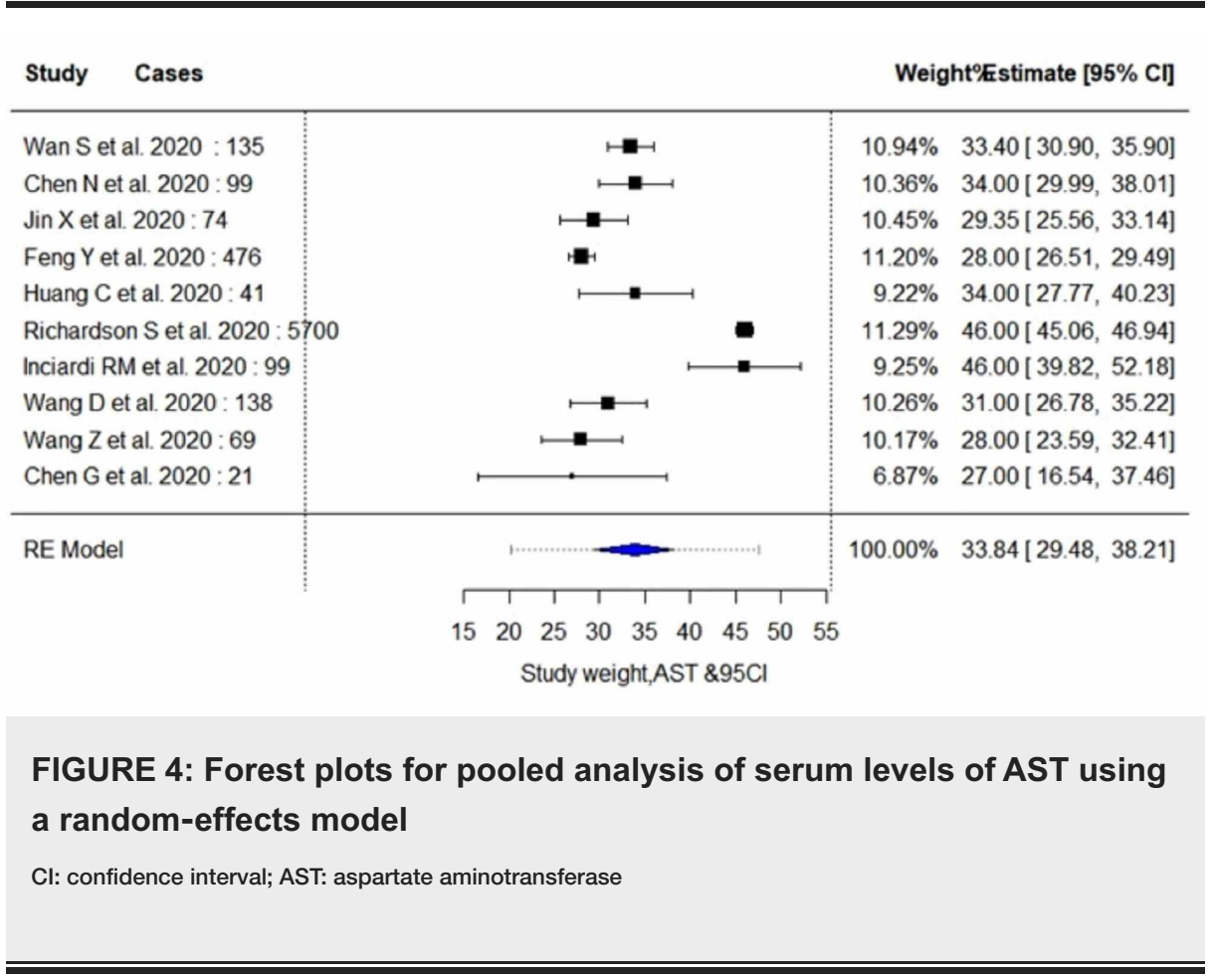




\section{Cureus}

\begin{tabular}{|c|c|c|}
\hline Cases & & Weight ${ }^{\circ}$ Fstimate $[95 \% \mathrm{Cl}]$ \\
\hline Wan S et al. $2020: 135$ & 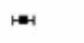 & $14.18 \% \quad 8.60[7.40,9.80]$ \\
\hline Jin X et al. $2020: 74$ & 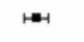 & $12.60 \% 10.00[8.62,11.38]$ \\
\hline Feng $Y$ et al. $2020: 476$ & a & $20.59 \% 10.10[9.56,10.64]$ \\
\hline Huang $C$ et al. $2020: 41$ & -1 & $13.72 \% \quad 11.70[10.45,12.95]$ \\
\hline Wang D et al. 2020 : 138 & $=$ & $17.07 \% \quad 9.80[8.90,10.70]$ \\
\hline Khamis F et al. $2020: 63$ & $\mapsto$ & $9.33 \% \quad 10.00[8.16,11.84]$ \\
\hline Chen G et al. $2020: 21$ & $=-1$ & $12.50 \% \quad 8.80[7.41,10.19]$ \\
\hline RE Model & mाImाmा & $100.00 \% \quad 9.87[9.15,10.60]$ \\
\hline
\end{tabular}

Study weight, Totalbilirubin $\& 95 \mathrm{Cl}$

FIGURE 5: Forest plots for pooled analysis of serum levels of total bilirubin using a random-effects model

$\mathrm{Cl}$ : confidence interval

\section{Subgroup analysis}

Six studies with 465 patients (severe cases, $n=131$; non-severe cases, $n=334$ ) were stratified based on the disease severity. Table 6 and Table 7 demonstrate the pooled estimates of LFTs of patients in the non-severe and severe group, respectively.

\begin{tabular}{|c|c|c|c|c|c|c|c|c|c|c|}
\hline \multirow{2}{*}{ Variable } & \multirow{2}{*}{$\mathbf{N}^{\mathbf{a}}$} & \multirow{2}{*}{ Estimate } & \multirow{2}{*}{$95 \% \mathrm{Cl}$} & \multirow{2}{*}{$\mathrm{N}^{\mathbf{b}}$} & \multirow{2}{*}{ Standard Error } & \multirow{2}{*}{ p-value } & \multicolumn{4}{|c|}{ Measure of Heterogeneity } \\
\hline & & & & & & & $\mathrm{T}^{2}$ & Q & $\mathrm{p}$ & $\mathrm{I}^{2}$ \\
\hline Albumin & 6 & 41.53 & $34.80-48.25$ & 334 & 3.4312 & $<0.0001$ & 45.98 & 182.65 & $<0.0001$ & $98.33 \%$ \\
\hline ALT & 6 & 21.60 & $18.70-24.51$ & 334 & 1.48 & $<0.0001$ & 5.80 & 9.21 & 0.0669 & $51.54 \%$ \\
\hline AST & 6 & 26.66 & 22.93-30.38 & 334 & 1.89 & $<0.0001$ & 14.38 & 20.44 & 0.0004 & $82.78 \%$ \\
\hline Total Bilirubin & 6 & 9.04 & 7.93-10.14 & 334 & 0.5618 & $<0.0001$ & 1.10 & 14.74 & 0.0053 & $73.32 \%$ \\
\hline
\end{tabular}

TABLE 6: Pooled estimates of abnormal liver biochemical indicators of patients in non-severe group

$\mathrm{Cl}$ : confidence interval; ALT: alanine aminotransferase; AST: aspartate aminotransferase

$\mathrm{N}^{\mathrm{a}}$ : number of studies; $\mathrm{N}^{\mathrm{b}}$ : number of patients 


\section{Cureus}

\begin{tabular}{|c|c|c|c|c|c|c|c|c|c|c|}
\hline \multirow{2}{*}{ Variable } & \multirow{2}{*}{$\mathbf{N}^{\mathbf{a}}$} & \multirow{2}{*}{ Estimate } & \multirow{2}{*}{$95 \% \mathrm{Cl}$} & \multirow{2}{*}{$\mathbf{N}^{\mathbf{b}}$} & \multirow{2}{*}{ Standard Error } & \multirow{2}{*}{ p-value } & \multicolumn{4}{|c|}{ Measure of Heterogeneity } \\
\hline & & & & & & & $\mathrm{T}^{2}$ & Q & $p$ & $I^{2}$ \\
\hline Albumin & 6 & 34.03 & $27.42-40.63$ & 131 & 0.2922 & $<0.0001$ & 43.66 & 66.11 & $<0.0001$ & $96.83 \%$ \\
\hline ALT & 6 & 31.66 & $25.07-38.25$ & 131 & 3.3641 & $<0.0001$ & 33.52 & 10.74 & 0.0567 & $55.64 \%$ \\
\hline AST & 6 & 41.79 & $32.85-50.72$ & 131 & 4.5584 & $<0.0001$ & 48.61 & 8.65 & 0.0704 & $51.43 \%$ \\
\hline Total Bilirubin & 6 & 9.97 & $8.46-11.48$ & 131 & 0.7715 & $<0.0001$ & 0.81 & 4.81 & $<0.0001$ & $98 \%$ \\
\hline
\end{tabular}

\section{TABLE 7: Pooled estimates of abnormal liver biochemical indicators of patients in severe group}

$\mathrm{CI}$, confidence interval; ALT, alanine aminotransferase; AST, aspartate aminotransferase

$\mathrm{N}^{\mathrm{a}}$, number of studies; $\mathrm{N}^{\mathrm{b}}$, number of patients

Serum Albumin

Of six studies included in the subgroup analysis, four studies with 258 subjects (severe cases, $\mathrm{n}=81$; nonsevere cases, $\mathrm{n}=177$ ) reported outcome data on serum albumin levels, as demonstrated in Table 6 and Table $7[3,7,12,14]$. The random-effect result of these studies demonstrated that patients in the severe group had lower serum levels for albumin in comparison to the non-severe individuals (weighted mean difference [WMD], $34.03 \mathrm{~g} / \mathrm{L}$; 95\% CI, 27.42 to 40.63; p<0.0001 vs WMD, $41.53 \mathrm{~g} / \mathrm{L}$; 95\% CI, 34.80 to 48.25; $\mathrm{p}<0.0001$ ). On analysis, both the subgroups yielded high heterogeneity $\left(\mathrm{I}^{2}=96.83 \%\right.$ vs $98.53 \%$; $\left.\mathrm{p}<0.0001\right)$.

Serum Alanine Aminotransferase (ALT)

All six studies with 468 subjects (severe cases, $n=131$; non-severe cases, $n=334$ ) included in the subgroup analysis reported outcome data on serum ALT levels, as demonstrated in Table 6 and Table 7 [3,7,12,14-16]. The random-effect result of these studies demonstrated that patients in the severe group had higher serum levels for ALT in comparison to the non-severe individuals (WMD, 31.66 U/L; 95\% CI, 25.07 to 38.25; p<0.0001 vs WMD, $21.60 \mathrm{U} / \mathrm{L} ; 95 \%$ CI, 18.70 to 24.51 ; $\mathrm{p}<0.0001)$. On analysis, both the subgroups yielded moderate heterogeneity $\left(\mathrm{I}^{2}=55.64 \% ; \mathrm{p}=0.0567\right.$ vs $^{2}=51.54 \%$; $\left.\mathrm{p}=0.0669\right)$.

Serum Aspartate Aminotransferase (AST)

Of six studies included in the subgroup analysis, five studies with 404 subjects (severe cases, $n=114$; nonsevere cases, $n=290$ ) reported outcome data on serum AST levels, as demonstrated in Table 6 and Table 7 [3,7,14-16]. The random-effect result of these studies demonstrated that patients in the severe group had higher serum levels for AST in comparison to the non-severe individuals (WMD, 41.79 U/L; 95\% CI, 32.85 to 50.72; $\mathrm{p}<0.0001$ vs WMD, $26.66 \mathrm{U} / \mathrm{L} ; 95 \% \mathrm{CI}, 22.93$ to 30.38; $\mathrm{p}<0.0001$ ). On analysis, the severe group yielded moderate heterogeneity $\left(\mathrm{I}^{2}=51.43 \% ; \mathrm{p}=0.0704\right)$, whereas the non-severe group yielded high heterogeneity $\left(\mathrm{I}^{2}=82.78 \% ; \mathrm{p}=0.0004\right)$.

Serum Total Bilirubin

Of six studies included in subgroup analysis, four studies with 335 subjects (severe cases, $\mathrm{n}=100$; non-severe cases, $\mathrm{n}=235$ ) reported outcome data on serum bilirubin levels, as demonstrated in Table 6 and Table 7 $[3,7,14,15]$. The random-effect result of these studies demonstrated that patients in the severe group had higher serum levels for total bilirubin in comparison to the non-severe individuals (WMD, $9.97 \mu \mathrm{mol} / \mathrm{L} ; 95 \%$ CI, 8.46 to 11.48 ; $\mathrm{p}<0.0001$ vs WMD, $9.04 \mu \mathrm{mol} / \mathrm{L}$; $95 \% \mathrm{CI}, 7.93$ to 10.14 ; $\mathrm{p}<0.0001$ ). On analysis, the severe group yielded high heterogeneity $\left(\mathrm{I}^{2}=98 \%\right.$; $\left.\mathrm{p}<0.0001\right)$, whereas the non-severe group yielded moderate heterogeneity $\left(\mathrm{I}^{2}=73.32 \%\right.$; $\left.=0.0053\right)$.

\section{Risk of bias and quality assessment}

Overall, six studies scored $\geqslant 5$ and were, therefore, regarded as high-quality publications. Four studies scored between 3 and 4 and were considered to be of medium-quality; the remaining two studies scored $<3$ and were considered to be of low-quality, as demonstrated in Table 8 . 


\section{Cureus}

\begin{tabular}{|c|c|c|c|c|c|c|c|c|c|c|c|c|}
\hline Criteria & $\begin{array}{l}\text { Wan } \\
\text { S et } \\
\text { al. } \\
{[14]}\end{array}$ & $\begin{array}{l}\text { Chen } \\
\mathrm{N} \text { et } \\
\text { al. } \\
\text { [1] }\end{array}$ & $\begin{array}{l}\text { Jin } \\
X \text { et } \\
\text { al. } \\
{[10]}\end{array}$ & $\begin{array}{l}\text { Feng } \\
\text { Y et } \\
\text { al. } \\
{[8]}\end{array}$ & $\begin{array}{l}\text { Huang } \\
\text { C et } \\
\text { al. [3] }\end{array}$ & $\begin{array}{l}\text { Richardson } \\
\text { S et al. [13] }\end{array}$ & $\begin{array}{l}\text { Inciardi } \\
\text { RM et } \\
\text { al. [9] }\end{array}$ & $\begin{array}{l}\text { Wang } \\
\text { D et } \\
\text { al. } \\
{[15]}\end{array}$ & $\begin{array}{l}\text { Khamis } \\
\text { F et al. } \\
\text { [11] }\end{array}$ & $\begin{array}{l}\text { Liu } \\
\text { J et } \\
\text { al. } \\
\text { [12] }\end{array}$ & $\begin{array}{l}\text { Wang } \\
Z \text { et } \\
\text { al. } \\
{[16]}\end{array}$ & $\begin{array}{l}\text { Chen } \\
\text { G et } \\
\text { al. } \\
{[7]}\end{array}$ \\
\hline $\begin{array}{l}\text { Representation of average adult in community } \\
\text { (population-based }=1 \text { point; } \text { multicenter }=0.5 \text { point; } \\
\text { single center }=0 \text { point) }\end{array}$ & 0 & 0 & 0.5 & 0.5 & 0 & 0.5 & 0 & 0 & 0.5 & 0 & 0 & 0 \\
\hline $\begin{array}{l}\text { Cohort size }(>100 \text { subjects }=1 \text { point; between } 50-99 \\
\text { subjects }=0.5 \text { point; }<50 \text { subjects }=0 \text { point) }\end{array}$ & 1 & 0.5 & 05 & 0.5 & 0 & 1 & 0.5 & 1 & 0.5 & 0.5 & 0.5 & 0 \\
\hline $\begin{array}{l}\text { Reported information on percentages and pattern of } \\
\text { liver injury (information with clarity }=1 \text { point; } \\
\text { information derived from percentages }=0.5 \text { point; } \\
\text { unclear }=0 \text { point) }\end{array}$ & 1 & 1 & 1 & 1 & 1 & 1 & 0 & 1 & 0 & 0 & 0 & 1 \\
\hline $\begin{array}{l}\text { Reported percentages of subjects with chronic liver } \\
\text { disease at baseline (yes }=1 \text { point; } n o=0 \text { point) }\end{array}$ & 1 & 1 & 1 & 1 & 1 & 1 & 0 & 1 & 0 & 0 & 0 & 1 \\
\hline $\begin{array}{l}\text { Assessed factors between mild and severe COVID- } \\
19 \text { (yes }=1 \text { point; no }=0 \text { point) }\end{array}$ & 1 & 0 & 0 & 0 & 1 & 0 & 0 & 1 & 0 & 1 & 0 & 1 \\
\hline $\begin{array}{l}\text { Adequate clinical assessment (yes }=1 \text { point; } n o=0 \\
\text { point) }\end{array}$ & 1 & 1 & 1 & 1 & 1 & 1 & 1 & 1 & 1 & 1 & 1 & 1 \\
\hline $\begin{array}{l}\text { Sufficient follow-up period for outcome to occur } \\
\text { (yes }=1 \text { point; unclear=0 point) }\end{array}$ & 1 & 1 & 0 & 1 & 1 & 1 & 0 & 0 & 0 & 1 & 1 & 0 \\
\hline $\begin{array}{l}\text { Adequate follow-up (all subjects were followed-up }=1 \\
\text { point; }>50 \% \text { subjects were followed-up }=0.5 \text { points; } \\
<50 \% \text { subjects were followed-up= } 0 \text { point) }\end{array}$ & 1 & 1 & 0 & 0 & 1 & 1 & 0 & 0 & 0 & 0 & 1 & 0 \\
\hline Total NOS Score & 7 & 5.5 & 4 & 5 & 6 & 6.5 & 1.5 & 5 & 2 & 3.5 & 3.5 & 4 \\
\hline
\end{tabular}

TABLE 8: Summary of quality assessment and risk of bias using the modified Newcastle-Ottawa scale (NOS)

\section{Discussion}

The present meta-analysis of 10 retrospective and two prospective studies investigated the possible link between impaired liver biochemistry and COVID-19 disease severity. Recent studies have shown that $37.2 \%$ to $76.3 \%$ of the patients infected with SARS-CoV-2 have impaired liver function [17-18].

In this meta-analysis, the laboratory findings revealed significantly lower levels of albumin and significantly higher levels of ALT and AST in COVID-19 patients; moreover, we also observed statistically insignificant higher levels of total bilirubin. Our results are in line with the previous researches on COVID-19, which also revealed hypoalbuminemia accompanied by elevated serum levels of aminotransferases and bilirubin as main indicators of liver injury [19-20]. The occurrence of hypoalbuminemia can be plausibly explained by the fact that albumin is a negative acute phase reactant, not a consequence of liver dysfunction.

The current suggests that critically ill COVID-19 positive individuals have a higher proportion of deranged liver biochemistries as compared to patients with a milder disease [21]. Upon comparing the LFTs of the severe and non-severe group, our results confirmed that patients with the severe clinical presentation of COVID-19 had lower levels of albumin and higher levels of total bilirubin, ALT, and AST relative to their counterparts. Recently, Guan et al. observed that approximately $28 \%$ and $56 \%$ of severely ill COVID- 19 patients had increased serum levels of ALT and AST, respectively; only $20 \%$ and $18 \%$ of the patients with non-severe COVID-19 showed higher serum ALT and AST levels, respectively [22]. The elevations, however, cannot be unequivocally linked to direct viral assault on the liver. However, evidence indicates that aminotransferases are a surrogate indicator of chronic inflammation and increased oxidative stress, which offers a possible explanation for their elevation during a viral illness [23].

Hepatic damage has been recognized as a significant prognostic factor for poorer outcomes of SARS-CoV and MERS-CoV infections. COVID-19 also causes liver injury; however, the exact mechanisms of liver injury remain unclear [5]. Recently, hepatic postmortem biopsies performed in deceased COVID-19 patients revealed moderate microvascular steatosis along with mild lobular and portal activity. The results were 
Four possible explanations of COVID-19-induced hepatic damage have currently been proposed. The first proposed mechanism is a direct attack on hepatocytes or biliary epithelium by SARS-CoV-2, leading to deranged LFTs. Both the liver and bile duct cells express angiotensin-converting enzyme 2 (ACE2) receptors that are the binding site for cellular entry by SARS-CoV-2 [5-6]. Although the direct entry of the virus into the hepatocytes via the ACE2 receptor appears to be the most logical explanation of liver injury, evidence suggests that hepatocytes do not express high levels of ACE2 receptors, making the liver an improbable target for infection. Additionally, a preliminary study by Chai et al. revealed a high level of ACE2 expression in cholangiocytes, implying that COVID-19-associated hepatic dysfunction may occur from injury to bile duct cells; however, consistency in the elevation of alkaline phosphatase (ALP) in COVID-19 patients has not been observed, further providing evidence against the proposed mechanism [25].

The second proposal suggests treatment side effects as a possible cause of hepatotoxicity in SARS-CoV-2 infection. Medicines like acetaminophen, hydroxychloroquine, and other antivirals that are commonly used against SARS infections may cause liver damage [5-6]. However, there is little evidence that drug combinations currently available to treat COVID-19 infection compromise liver function. Fan et al. conducted a study on 148 patients with COVID-19-associated liver damage. The authors proposed that liver dysfunction may be due to the antivirals, lopinavir/ritonavir, used to treat SARS-CoV-2 infection [17]. Furthermore, results by Cai et al. also indicate that the hepatic damage seen in hospitalized patients with COVID-19 may be due to the adverse effects of drugs. They also found that patients with deranged LFTs were at a greater risk of severe disease progression [18].

The third mechanism suggested pertains to immune-mediated liver damage. There is a possibility that the hyperactivated defense system of the body in response to the virus may lead to the development of a cytokine storm and subsequent liver impairment [6,26]. Lastly, it has been speculated that SARS-CoV-2 may worsen already-compromised hepatic function in patients with pre-existing liver diseases such as viral hepatitis $[5,26]$.

Here, it is also imperative to shed light on other causes of liver damage such as hepatic hypoxia due to pulmonary insufficiency, pulmonary embolism, low cardiac output, and cerebral respiration insufficiency. Hypoxic hepatitis, also referred to as shock liver and ischemic hepatitis is attributed to systemic hypotension or hypoxemia, resulting in decreased blood flow to the hepatocytes [27]. It is pre-established that patients with a severe presentation of COVID-19 develop pneumonia with subsequent progression to acute respiratory distress syndrome, septic shock, and ultimately death [3]. In addition, the occurrence of pulmonary embolism has been identified in some patients with SARS-COV-2 infection, resulting in acute respiratory insufficiency [27-28]. Thus, hepatic injury in COVID-19 could be plausibly explained by the fact that respiratory insufficiency $(\mathrm{PaO} 2<45 \mathrm{mmHg}$ ) could lead to liver injury due to reduced oxygen supply to the hepatocytes, which may eventually progress to liver failure.

Although SAR-COV-2 infection mainly attacks the respiratory system, invasion of the cardiovascular system by the virus is not uncommon. A retrospective study conducted in China among patients who died of COVID-19 revealed cardiac damage in $89 \%$ and cardiac failure in $14.6 \%$ of the patients [20]. The study also revealed that cardiac failure was the third most common cause of death after acute respiratory failure and sepsis syndrome/MOF [20]. Low cardiac output secondary to cardiac failure, as seen in respiratory disease and septic shock, may also predispose to liver injury and hypoxic hepatitis. The primary pathophysiology involves hepatic congestion from right heart failure along with reduced blood flow to the liver or reperfusion injury following ischemia [27]. Evidence suggests that sepsis in COVID-19 contributes to hypoxic liver injury, causing a surge in liver biomarkers, which plausibly explains the higher levels of serum ALT, AST, and total bilirubin in severe COVID-19 patients relative to the non-severe group in our study [18,29]. Nonetheless, the involvement of the brainstem by SARS-COV-2 may also affect the respiratory center, causing breathing difficulties and subsequent hypoxemia, further leading to hepatic hypoxia and ischemia in critically ill COVID-19 patients [30]. Hence COVID-19 encompasses an array of problems that could result in liver injury either by directly infecting the liver or causing damage secondary to the involvement of other systems such as the pulmonary, cardiovascular, or neurological system. However, there is still a scarcity of data reporting liver failure in COVID-19 patients with chronic liver diseases. Future studies are warranted to explore the mechanisms of hepatic dysfunction in patients with COVID-19.

The present meta-analysis, however, has a few limitations. Firstly, most of the studies included in the metaanalysis had a retrospective study design; thus, there is a danger of bias in data collection. Second, since all the studies reported only hospitalized patients, the occurrence of liver injury among COVID-19 patients in our study may have been overestimated. Therefore, our results cannot be extrapolated to the entire population of SARS-CoV-2 patients. Third, many studies reported patients with pre-existing chronic liver disease, which renders them susceptible to developing an acute liver injury. However, the present analysis did not monitor the possible effects of potential confounders, such as age, gender, and comorbidities; hence, the findings must be interpreted with caution. Moreover, the majority of the studies included is from China, and, thus, may not represent variations between different populations. 
Despite, the above limitations, this systematic review and meta-analysis provide useful information on the prevalence and liver complications of COVID-19 infection.

\section{Conclusions}

This review comprehensively analyzes the liver enzymes of COVID-19 patients who experienced liver injuries. It also correlates deranged liver biomarkers with the severity of the COVID-19 disease. The analysis revealed that liver function derangements, such as hypoalbuminemia, hyperbilirubinemia, and elevated aminotransferase levels, are common in COVID-19 infection. Moreover, these abnormalities were found to be relatively higher in severe cases of COVID-19 than in non-severe cases. Hence, we conclude that impaired liver biochemistry serves as a prognostic factor to assess COVID-19 severity. Liver markers should, therefore, be observed and monitored continuously to avoid poor outcomes.

\section{Additional Information}

\section{Disclosures}

Human subjects: All authors have confirmed that this study did not involve human participants or tissue. Animal subjects: All authors have confirmed that this study did not involve animal subjects or tissue. Conflicts of interest: In compliance with the ICMJE uniform disclosure form, all authors declare the following: Payment/services info: All authors have declared that no financial support was received from any organization for the submitted work. Financial relationships: All authors have declared that they have no financial relationships at present or within the previous three years with any organizations that might have an interest in the submitted work. Other relationships: All authors have declared that there are no other relationships or activities that could appear to have influenced the submitted work.

\section{Acknowledgements}

The authors would like to thank Mr. Ahmed Miftah, Department of Civil Engineering, Cyprus International University, and Department of Biochemistry, Faculty of Medicine, University of Benghazi, Benghazi, Libya.

\section{References}

1. Chen N, Zhou M, Dong X, et al.: Epidemiological and clinical characteristics of 99 cases of 2019 novel coronavirus pneumonia in Wuhan, China: a descriptive study. Lancet. 2020, 395:507-513. 10.1016/S01406736(20)30211-7

2. Coronavirus disease (COVID-19) pandemic . (2020). Accessed: June 20, 2020: https://www.who.int/emergencies/diseases/novel-coronavirus-2019? gclid=EAIaIQobChMI5aeVt7rp6gIVGYXVCh2CsQWZEAAYASAAEgL....

3. Huang C, Wang Y, Li X, et al.: Clinical features of patients infected with 2019 novel coronavirus in Wuhan, China. Lancet. 2020, 395:497-506. 10.1016/S0140-6736(20)30183-5

4. Kunutsor SK, Laukkanen JA: Cardiovascular complications in COVID-19: a systematic review and metaanalysis. J Infect. 2020, 81:e139-e141. 10.1016/j.jinf.2020.05.068

5. Xu L, Liu J, Lu M, Yang D, Zheng X: Liver injury during highly pathogenic human coronavirus infections . Liver Int. 2020, 40:998-1004. 10.1111/liv.14435

6. Guan GW, Gao L, Wang JW, et al.: Exploring the mechanism of liver enzyme abnormalities in patients with novel coronavirus-infected pneumonia [Article in Chinese]. Chin J Hepatol. 2020, 28:100-106. 10.3760/cma.j.issn.1007-3418.2020.02.002

7. Chen G, Wu D, Guo W, et al.: Clinical and immunological features of severe and moderate coronavirus disease 2019. J Clin Invest. 2020, 130:2620-2629. 10.1172/JCI137244

8. Feng Y, Ling Y, Bai T, et al.: COVID-19 with different severities: a multicenter study of clinical features . Am J Respir Crit Care Med. 2020, 201:1380-1388. 10.1164/rccm.202002-04450C

9. Inciardi RM, Adamo M, Lupi L, et al.: Characteristics and outcomes of patients hospitalized for COVID-19 and cardiac disease in Northern Italy. Eur Heart J. 2020, 41:1821-1829. 10.1093/eurheartj/ehaa388

10. Jin X, Lian JS, Hu JH, et al.: Epidemiological, clinical and virological characteristics of 74 cases of coronavirus-infected disease 2019 (COVID-19) with gastrointestinal symptoms. Gut. 2020, 69:1002-1009. 10.1136/gutjnl-2020-320926

11. Khamis F, Al-Zakwani I, Al Naamani H, et al.: Clinical characteristics and outcomes of the first 63 adult patients hospitalized with COVID-19: an experience from Oman. J Infect Public Health. 2020, 13:906-913. 10.1016/j.jiph.2020.06.002

12. Liu J, Liu Y, Xiang P, et al.: Neutrophil-to-lymphocyte ratio predicts critical illness patients with 2019 coronavirus disease in the early stage. J Transl Med. 2020, 18:206. 10.1186/s12967-020-02374-0

13. Richardson S, Hirsch JS, Narasimhan M, et al.: Presenting characteristics, comorbidities, and outcomes among 5700 patients hospitalized with COVID-19 in the New York City area. JAMA. 2020, 323:2052-2059. 10.1001/jama.2020.6775

14. Wan S, Xiang Y, Fang W, et al.: Clinical features and treatment of COVID-19 patients in northeast Chongqing. J Med Virol. 2020, 92:797-806. 10.1002/jmv.25783

15. Wang D, Hu B, Hu C, et al.: Clinical characteristics of 138 hospitalized patients with 2019 novel coronavirus-infected pneumonia in Wuhan, China. JAMA. 2020, 323:1061-1069. 10.1001/jama.2020.1585

16. Wang Z, Yang B, Li Q, Wen L, Zhang R: Clinical features of 69 cases with coronavirus disease 2019 in Wuhan, China. Clin Infect Dis. 2020, 71:769-777. 10.1093/cid/ciaa272

17. Fan Z, Chen L, Li J, et al.: Clinical features of COVID-19-related liver functional abnormality . Clin Gastroenterol Hepatol. 2020, 18:1561-1566. 10.1016/j.cgh.2020.04.002 
18. Cai Q, Huang D, Yu H, et al.: COVID-19: abnormal liver function tests. J Hepatol. 2020, 73:566-574. 10.1016/j.jhep.2020.04.006

19. Huang Y, Yang R, Xu Y, Gong P: Clinical characteristics of 36 non-survivors with COVID-19 in Wuhan, China (PREPRINT). medRxiv. 2020, 10.1101/2020.02.27.20029009

20. Zhang B, Zhou X, Qiu Y, et al.: Clinical characteristics of 82 death cases with COVID-19 [PREPRINT] . medRxiv. 2020, 10.1101/2020.02.26.20028191

21. Hu LL, Wang WJ, Zhu QJ, Yang L: Novel coronavirus pneumonia-related liver injury: etiological analysis and treatment strategy [Article in Chinese]. Chin J Hepatol. 2020, 28:97-99. 10.3760/cma.j.issn.10073418.2020.02.001

22. Guan WJ, Ni ZY, Hu Y, et al.: Clinical characteristics of coronavirus disease 2019 in China . N Engl J Med. 2020, 382:1708-1720. 10.1056/NEJMoa2002032

23. Yamada J, Tomiyama H, Yambe M, et al.: Elevated serum levels of alanine aminotransferase and gamma glutamyltransferase are markers of inflammation and oxidative stress independent of the metabolic syndrome. Atherosclerosis. 2006, 189:198-205. 10.1016/j.atherosclerosis.2005.11.036

24. Xu Z, Shi L, Wang Y, et al.: Pathological findings of COVID-19 associated with acute respiratory distress syndrome. Lancet Respir Med. 2020, 8:420-422. 10.1016/S2213-2600(20)30076-X

25. Chai X, Hu L, Zhang Y, et al.: Specific ACE2 expression in cholangiocytes may cause liver damage after 2019-nCoV infection [PREPRINT]. bioRxiv. 2020, 10.1101/2020.02.03.931766

26. Li J, Fan JG: Characteristics and mechanism of liver injury in 2019 coronavirus disease . J Clin Transl Hepatol. 2020, 8:13-17. 10.14218/JCTH.2020.00019

27. Aslan S, Meral M, Akgun M, Acemoglu H, Ucar EY, Gorguner M, Mirici A: Liver dysfunction in patients with acute pulmonary embolism. Hepatol Res. 2007, 37:205-213. 10.1111/j.1872-034X.2007.00014.X

28. Bompard F, Monnier H, Saab I, et al.: Pulmonary embolism in patients with COVID-19 pneumonia . Eur Respir J. 2020, 56:2001365. 10.1183/13993003.01365-2020

29. Phipps MM, Barraza LH, LaSota ED, et al.: Acute liver injury in COVID-19: prevalence and association with clinical outcomes in a large US cohort. Hepatology. 2020, 72:807-817. 10.1002/hep.31404

30. Gandhi S, Srivastava AK, Ray U, Tripathi PP: Is the collapse of the respiratory center in the brain responsible for respiratory breakdown in COVID-19 patients?. ACS Chem Neurosci. 2020, 11:1379-1381. 10.1021/acschemneuro.0c00217 